Zongyu Jiang

Yun Gao

Jie Liu

http://dx.doi.org/10.21278/brod70209

ISSN 0007-215X

eISSN 1845-5859

\title{
A LID APPROACH FOR PREDICTING WAVE INDUCED MOTIONS OF TRIMARAN IN REGULAR WAVES
}

UDC 629.5.022.3:629.5.017

Professional paper

\begin{abstract}
Summary
The wave induced motions of a trimaran sailing in regular head waves were predicted by using the three dimensional boundary integral method. Large wave elevation on the free surface in between the trimaran's main-hull and the outriggers occurred at some specific frequencies in the numerical model. The large wave elevation also induced large heave and pitch motions of trimaran. However, the large wave elevation and corresponding large motions were not observed during towing tank tests. A lid approach was introduced in this paper by placing lid on the free surface in between hulls to suppress the unrealistically large wave elevation and to correctly predict the induced motions of trimaran. The feasibility and practicability of lid approach were validated against experimental results.
\end{abstract}

Key words: $\quad$ Trimaran; lid approach; ship motions; wave elevation; regular waves

\section{Introduction}

Naval architects have been pursuing one permanent goal - achieving maximum speed with minimum energy. Many distinct hull forms have been created and innovated over several decades. Among them is the trimaran hull, which held the attention of ship designers and researchers immediately after the concept was proposed. The main-hull of a trimaran could be constructed much slenderer than a regular mono-hull vessel to reduce drag. Meanwhile the trimaran could exhibit excellent seakeeping performance with outriggers. RV Triton, launched in May 2000 and delivered in August 2000, is the world's first large motor powered trimaran, with a displacement of $1200 \mathrm{t}$, a length of $90 \mathrm{~m}$ and a beam of $22.5 \mathrm{~m}$ [1]. The successful trials program proved that the design was able to satisfy any operational requirement in the exactly same way as a mono-hull vessel. Significant drag reduction leading to high-speed has been well demonstrated through this program. Hitherto, multiple types of trimaran have been enlisted in various fields, like Benchijigua Express and Independence class LCS.

A number of research papers focusing on seakeeping performance of trimaran have been published by various researchers. Bingham et al. [2] computed motions (heave and pitch) and loads of a trimaran in regular waves using frequency-domain boundary integral method. They used two methods, pulsating and translating source and purely pulsating source distributions on 
mean wetted surface. The results obtained from the two approaches show that the latter is surprisingly more suitable for fast vessels. Model test is an efficient method to research wave induced motions [3] and consequently many researchers employ this method to study the motions of trimaran. Hebblewhite, Sahoo and Doctors [4] investigated the effects of longitudinal stagger of outriggers on heave and pitch motions of a typical trimaran hull. A round-bilge high-speed hull form was constructed and four different longitudinal stagger positions were tested in the experiments. Hebblewhite, Sahoo and Doctors [4] also provided numerical results obtained from code HYDROS. The paper stated that the variation in the radius of gyration could have a significant effect on the heave and pitch motions. McDonald et al. [5] compared trimaran small waterplane area center hull, monohull and trimaran hullforms from the angle of design. Pavkov and Morabito [6] conducted a series of tests of two different trimaran models to determine the effect of finite water depth on the resistance, heave and trim.

In this paper, the three-dimensional boundary integral method (BIM) has been utilized to analyze wave induced motions of a trimaran hull in regular head waves. In the numerical model, large wave amplitude of the free-surface in between the main-hull and outriggers were observed in some specific narrow frequency bands. The large wave elevation also resulted in unrealistically large hull motion in heave and pitch. However, this phenomenon of large wave elevation and large hull motion at specific frequencies were not observed during towing tank experiments. The unrealistic phenomenon has also been reported in literatures with regard to numerical simulations of multiple floating bodies and catamarans ([7], [8]). Researchers also provided some practical methods to solve this issue, such as directly introducing artificial viscosity to the free surface [8] and adding artificial damping with a rigid lid [9] or with a flexible lid [10]. However, most researches focused on the multiple floating structures with zero velocity. This was different from the situation happening to the trimaran because the large wave elevation only happened when the velocity was larger than zero according to our research.

This paper introduces the lid approach to the prediction of wave induced motions of trimaran. A rigid lid with artificial damping was laid on the free surface in between the mainhull and outriggers to suppress the unrealistic wave elevation. The results showed that the lid approach successfully eliminated the negative effect of large wave elevation around the trimaran. The lid approach removed the peak of Response Amplitude Operator (RAO) of hull motion at frequencies where the large wave elevation occurred. Furthermore, this approach hardly influenced the RAO value at another frequencies. To validate the lid approach, a series of tests were also carried out in the towing tank of Dalian University of Technology. The agreements of numerical prediction and experimental data indicated that the lid approach could be a satisfying practical solution.

\section{The trimaran model}

The trimaran model was composed of one central main-hull and two outriggers standing respectively on each side of the main-hull. All these three hulls were rigidly connected together as an entire floating structure with two wooden beams. As a consequence, the trimaran model exactly held six degrees of freedom though it was composed of three separate hulls. The mainhull was of hard chine type, with a transom stern designed as a high-speed displacement hull form. The outriggers, which were approximately a quarter in length of the main-hull, had similar hull lines to those of main-hull. Nevertheless, the main dimensions of outrigger were not on the same scale to those of main-hull. The hull lines and the main dimensions of the trimaran are shown in Fig. 1 and Table 1, respectively. 
Table 1 Main dimension

\begin{tabular}{ccc}
\hline Item & Main-hull's & Outrigger's \\
\hline Waterline length $(\mathrm{m})$ & 4.0 & 1.0 \\
Waterline beam $(\mathrm{m})$ & 0.3584 & 0.085 \\
Draft $(\mathrm{m})$ & 0.17 & 0.10 \\
Displacement mass $(\mathrm{kg})$ & 129.07 & 4.45 \\
Wetted surface area $\left(\mathrm{m}^{2}\right)$ & 1.899 & 0.201 \\
Block coefficient $\mathrm{C}_{\mathrm{B}}$ & 0.530 & 0.524 \\
Prismatic coefficient $\mathrm{C}_{\mathrm{p}}$ & 0.769 & 0.746 \\
\hline
\end{tabular}
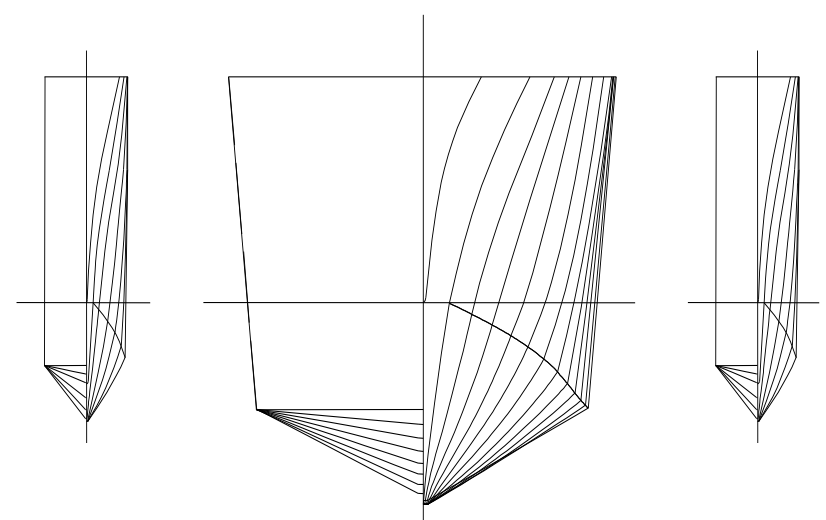

Fig. 1 Trimaran bodyline

Different schemes were designed in accordance with changes of longitudinal and/or transverse positions of the outriggers. The positions of the outriggers and the coordinate system are displayed in Fig. 2. Here, $O$, intersection of central longitudinal section and midship section on waterplane, represents original point; $a$ represents the distance from midship section of the main-hull to that of the outriggers, positive towards the bow; $b$ denotes the distance from central longitudinal section of main-hull to that of outriggers. As shown in Table 2, four schemes are used as sample to illustrate the phenomenon of unrealistic wave elevation: Scheme 4-13, Scheme 4-07, Scheme 5-13 and Scheme 5-07. Scheme 4-13 and Scheme 4-07 are selected to validate the lid approach's feasibility.

Table 2 Location of outrigger

\begin{tabular}{ccc}
\hline Scheme & $a(\mathrm{~m})$ & $b(\mathrm{~m})$ \\
\hline $4-13$ & -1.3 & 0.4 \\
$4-07$ & -0.7 & 0.4 \\
$5-13$ & -1.3 & 0.5 \\
$5-07$ & -0.7 & 0.5 \\
\hline
\end{tabular}




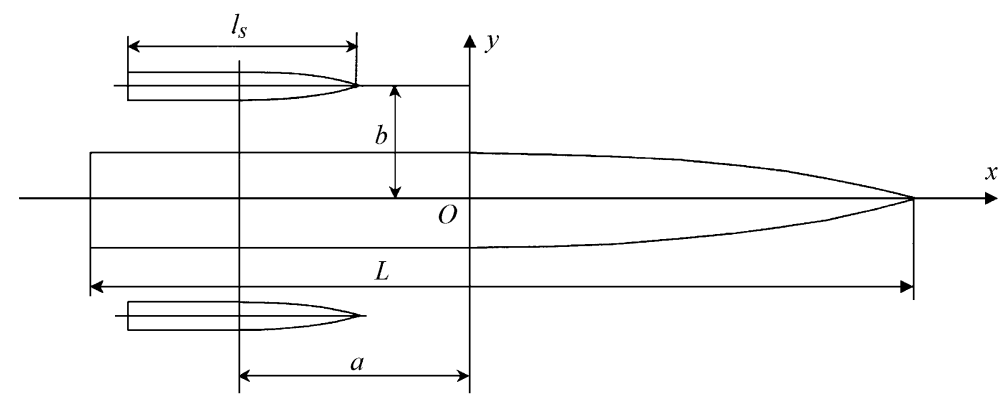

Fig. 2 Position of outriggers and coordinate system

All calculations were carried out in deep water condition in head seas with three speeds: $1.464 \mathrm{~m} / \mathrm{s}, 3.091 \mathrm{~m} / \mathrm{s}$ and $4.392 \mathrm{~m} / \mathrm{s}$. The corresponding Froude Numbers are 0.23, 0.49, and 0.70 . The wave frequency ranges from $0.4 \mathrm{rad} / \mathrm{s}$ to $6 \mathrm{rad} / \mathrm{s}$.

Table 3 Wave lengths and corresponding wave frequencies

\begin{tabular}{|c|c|c|c|c|c|c|c|c|c|c|}
\hline Item & \multicolumn{10}{|c|}{ Value } \\
\hline Wave length (m) & 12 & 11 & 9 & 8 & 7 & 6 & 5 & 4 & 3 & 2 \\
\hline Wave frequencies $(\mathrm{rad} / \mathrm{s})$ & 2.3 & 2.4 & 2.6 & 2.8 & 3.0 & 3.2 & 3.5 & 3.9 & 4.5 & 5.5 \\
\hline
\end{tabular}

The research also applied towing tank test results to validate the computational results. The wave direction and travelling speeds were the same with numerical prediction but the tests were carried out with respect to wave length. In order to conveniently compare, the wave lengths employed in the tests are translated into circular frequencies, which are presented in Table 3.

\section{Computational methodology and numerical model}

The Cartesian coordinate system $\boldsymbol{X}=(x, y, z)$ is utilized by considering the plane $z=0$ the undisturbed free surface of water and the space $z<0$ the fluid domain. The fluid is assumed to be incompressible, inviscid, and the flow is irrotational. As a result, the velocity vector $V$ can be represented by the gradient of the potential $\phi$

$$
V=\nabla \phi
$$

Therefore, the governing partial differential equation can be described

$$
\frac{\partial^{2} \phi}{\partial x^{2}}+\frac{\partial^{2} \phi}{\partial y^{2}}+\frac{\partial^{2} \phi}{\partial z^{2}}=0
$$

A trimaran exhibits 6 degrees of freedom so it could be assumed as a rigid body by means of a mass-spring system. As a consequence, a trimaran's motions induced by waves in the frequency domain can be described with the following equation:

$$
\sum_{j=l}^{6}\left(M_{k j}+a_{k j}\right) \ddot{x}_{j}+b_{k j} \dot{x}_{j}+c_{k j} x_{j}=F_{k} \quad k=1,2, \ldots, 6
$$

where:

$k, j=$ suffixes describing hydrodynamic property in $k$ mode as a result of motion in $j$ mode $M_{k, j}=$ mass of trimaran 
$a_{k, j}=$ added mass matrix

$b_{k, j}=$ damping coefficient matrix

$c_{k, j}=$ hydrostatic restoring matrix

$F_{k}=$ external force in mode $k$

According to the potential flow theory, the added mass and damping coefficient can be described

$$
\begin{aligned}
& a_{k, j}=\operatorname{Re}\left[\rho \int_{S} \phi_{j} n_{k} d S\right] \\
& b_{k, j}=\operatorname{Im}\left[\rho \int_{S} \phi_{j} n_{k} d S\right] \omega
\end{aligned}
$$

where

$S=$ body surface

$\rho=$ density of water

$\omega=$ wave frequency

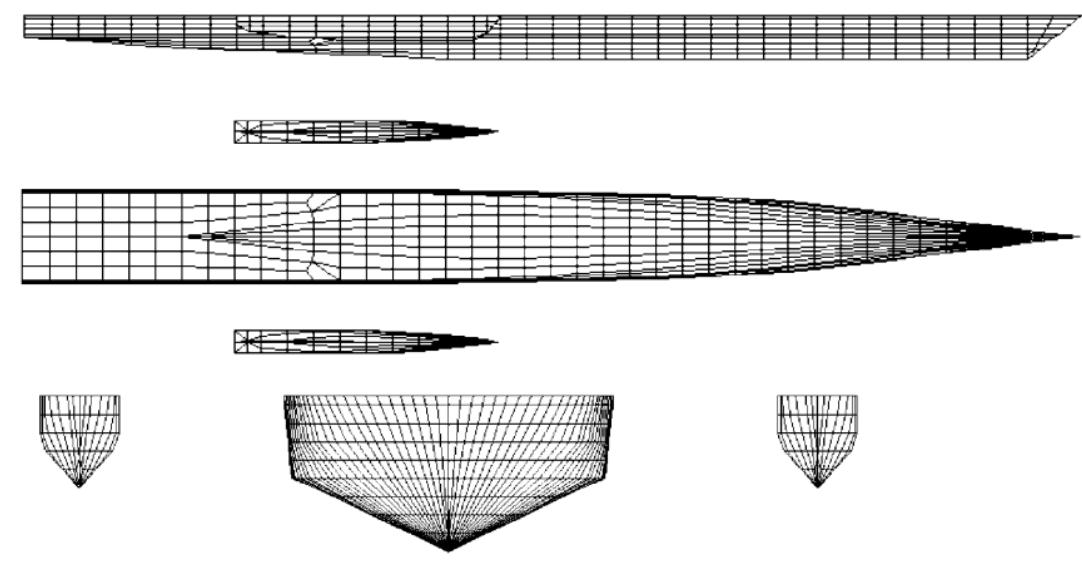

Fig. 3 Panel distribution (top, side view; middle, top view; bottom, front view)

The boundary integral method (BIM) was employed to predict wave induced motions of the trimaran. The fundamentals of this method lie in the Green's theorem, in which velocity potential at any point of the fluid domain is represented by distributions of singularities on boundary surfaces ([11], [12], [13]). Consequently, the wetted surface of body should be represented by a number of panels. Figure 3 shows the distribution of panels of Scheme 4-07 as an example. The mesh convergence tests were performed to establish a proper mesh for the calculation by using the Scheme 4-13. During the tests, the body surface was respectively represented with 229, 980, 1738 and 4848 meshes. The results, as shown in Fig. 4, indicated that the calculation converged. When the body surface was built with 1738 meshes, the calculation reaches a fairly good precision. Thus, the model with 1738 meshes was employed for the calculation. 


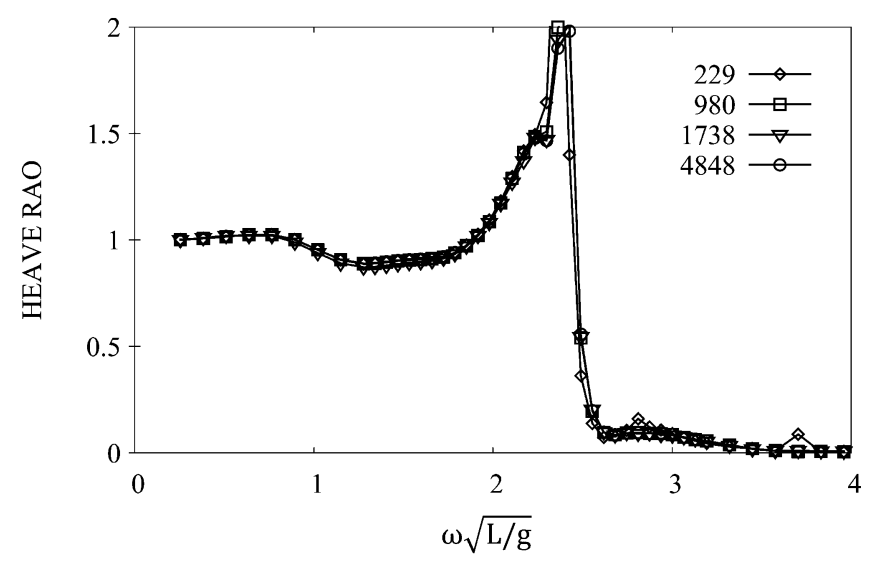

Fig. 4 Convergence test, $4-13, \mathrm{Fn}=0.49$

The boundary-integral equations of wave problems suffer an irregular frequency issue. The irregular frequencies correspond to the eigen frequencies of the homogeneous Dirichlet problem arising from the existence of fluid and free surface inside the body [14]. Consequently, numerical solutions of the integral equations in the vicinity of these irregular frequencies are incorrect. In order to eliminate irregular frequencies, the technique proposed by Ohmatsu [15] is employed. By imposing an extra boundary condition on the interior of the free surface, the Green's integral equation is extended to the interior of the free surface ([16], [17], [18]). The encounter frequency method was used to perform the calculation. This method is widely used for calculating hydrodynamics of body with forward speed and it can provide reasonable results ([19], [20]). The relationship between the encounter frequency and wave frequency follows

$$
\omega_{e}=\omega-U k \cos \theta
$$

where

$$
\begin{aligned}
& \omega_{e}=\text { encounter frequency } \\
& \omega=\text { wave frequency } \\
& U=\text { ship velocity } \\
& k=\text { wave number } \\
& \theta=\text { the direction of wave propagation relative to the } \mathrm{x} \text {-axis }
\end{aligned}
$$

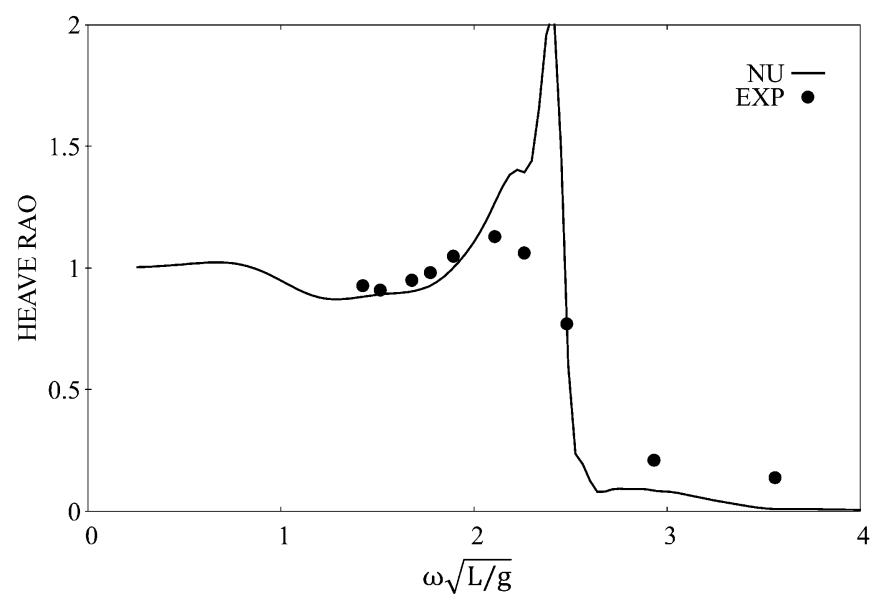

Fig. 5 Abnormal resonant peak of heave RAO curve, 4-13, Fn=0.49 


\section{The phenomenon of unrealistic wave elevation and lid approach}

\subsection{The unrealistic wave elevation}

In the process of calculating the trimaran's motions in waves, a very interesting phenomenon was discovered, which was somewhat like the irregular frequency issue, even though extension for source distribution in the interior of the free surface of the hull is imposed to eliminate irregular frequencies. This phenomenon occurred at some specific frequencies and there also existed abnormal resonance peak in the computed RAO curve in the vicinity. It only happened in numerical simulation and had never been observed during tests. One numerical example and its comparison to the experimental result are presented in Fig. 5 where the RAOs are plotted with line representing numerical data and dark points representing experimental data. It was apparent that the computed result deviate significantly from the experimental data at nondimensional frequency 2.4 (wave frequency $3.8 \mathrm{rad} / \mathrm{s}$ ) and its neighbourhood. Further investigation revealed that, at the specific frequencies, wave amplitudes of the free surface in between the main-hull and the outriggers were unrealistically large. The abnormal elevation of the free surface in between the main-hull and the outrigger is shown in Fig. 6. The peak locates at nondimensional frequency 2.4 as well. The added mass and radiation damping coefficient are shown in Fig. 7. There existed a dent on the curve of added mass and the curve of radiation damping precipitates in the vicinity of nondimensional frequency 2.4. According to equation (3), the small value of added mass and radiation damping could lead to large motions. Therefore, the large heave motion of hull was induced by the unrealistic large wave elevation in the gap between hulls.

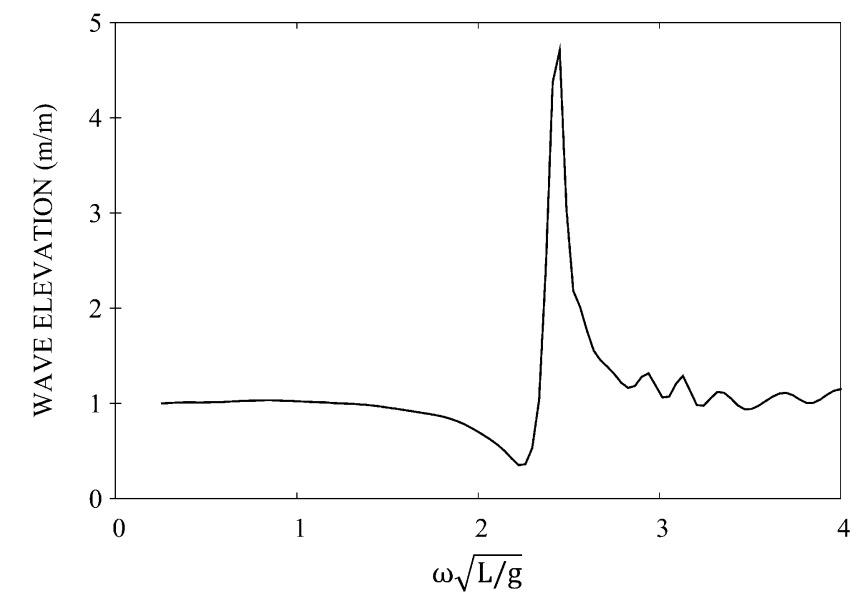

Fig. 6 RAO of free surface elevation in between hulls, 4-13, Fn=0.49 $(x=-0.9 m, y=0.27 \mathrm{~m})$ 


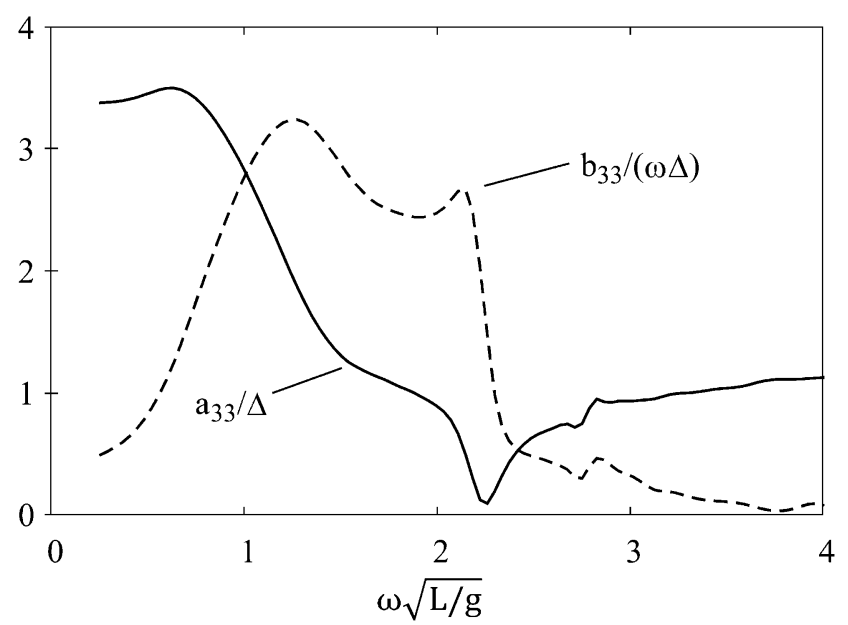

Fig. 7 Added mass and radiation damping, 4-13, $\mathrm{Fn}=0.49$ ( $\Delta$ denotes the displacement mass)

The occurring frequency of large wave elevation was connected to forward speed and configuration of gaps in between the main-hull and the outriggers. Firstly, the phenomenon occurred at a certain encounter frequency when the configuration of gap was fixed. Examples of occurring frequencies and their corresponding encounter frequencies are shown in Table 4. Secondly, the encounter frequency of resonance was inversely proportional to the gap width. The encounter frequency of Scheme 4-07 and Scheme 4-13 is approximately $8.2 \mathrm{rad} / \mathrm{s}$ and the encounter frequency of Scheme 5-07 and Scheme 5-13 was approximately $7.8 \mathrm{rad} / \mathrm{s}$.

Table 4 Occurring frequencies and corresponding encounter frequencies

\begin{tabular}{cccc}
\hline Scheme & Froude number & $\begin{array}{c}\text { Resonance freq. } \\
(\mathrm{rad} / \mathrm{s})\end{array}$ & $\begin{array}{c}\text { Encounter freq. } \\
(\mathrm{rad} / \mathrm{s})\end{array}$ \\
\hline \multirow{4}{*}{$4-13$} & 0.23 & 4.8 & 8.2 \\
& 0.49 & 3.8 & 8.4 \\
& 0.7 & 3.3 & 8.2 \\
$4-07$ & 0.23 & 4.7 & 8.1 \\
& 0.49 & 3.7 & 8.2 \\
& 0.7 & 3.3 & 8.2 \\
$5-13$ & 0.23 & 4.6 & 7.8 \\
& 0.49 & 3.6 & 7.7 \\
& 0.7 & 3.2 & 7.8 \\
$5-07$ & 0.23 & 4.6 & 7.8 \\
& 0.49 & 3.6 & 7.7 \\
\hline
\end{tabular}

Molin [21] investigated the piston mode motion of water occurs in the moonpool. According to his theory, the natural frequency of piston mode motion can be described

$$
\omega_{n}=\sqrt{\frac{g}{h(1+C)}}
$$

with

$$
C=\frac{1}{2 \pi} \frac{1}{b l h}\left[b^{2} l \arg \sinh \frac{l}{b}+b l^{2} \arg \sinh \frac{b}{l}+\frac{1}{3}\left(b^{3}+l^{3}\right)-\frac{1}{3}\left(b^{2}+l^{2}\right)^{3 / 2}\right]
$$


where

$$
\begin{aligned}
& g=\text { gravity acceleration } \\
& h=\text { draft of moonpool } \\
& b=\text { width of moonpool } \\
& l=\text { length of moonpool }
\end{aligned}
$$

While considering the occurring frequency as the natural frequency of piston mode and using this equation to calculate the occurring frequency, $h$ was the draft of hard chine of outrigger, $b$ was the gap width at the midship section of outrigger and $l$ was equal to the length of outrigger. Consequently, the gap configuration of Scheme 4-13 was similar to that of Scheme 4-07 and the condition between Scheme 5-13 and 5-07 was alike. According to Molin's equation, the resonant frequency of Scheme 4-13 and 4-07 is $8.2 \mathrm{rad} / \mathrm{s}$ and the resonant frequency of Scheme 5-13 and 5-07 is 7.4 rad/s. These frequencies slightly deviated from those listed in Table 4. Molin's theory is based on an assumption that the moonpool is rectangular. However, the gap between hulls is not strictly rectangular because of the hull line. As a result, the natural frequency derived from Molin's equation slightly deviated from the numerical calculation.

\subsection{The lid approach}

In order to suppress the large wave elevation, a lid was utilized to replace the two physical free surfaces in between the main-hull and the outriggers. One exemplary outline of the lid approach was given in Fig. 8. Only a replacement of sufficient part of resonant free surface was capable to achieve satisfactory results so the lid was arbitrarily rectangular. This lid was independent of the trimaran so the lid and trimaran composed a multi-body system which involves twelve degrees of freedom due to rigid-body assumption. The motion equation for multi-body system in regular waves ([22], [23]) can be described as:

$$
\sum_{n=1}^{M} \sum_{j=1}^{6}\left(\delta_{n m} M_{k j}^{n m}+a_{k j}^{n m}\right) \ddot{x}_{j}^{m}+b_{k j}^{n m} \dot{x}_{j}^{m}+\delta_{n m} c_{k j}^{n m} x_{j}^{m}=F_{k}^{n} \quad n=1,2, \ldots, M \quad k=1,2, \ldots, 6
$$

where:

$M=$ the number of bodies, obviously, $M=2$ in this case

$\delta_{n m}=$ the Kroneker delta function

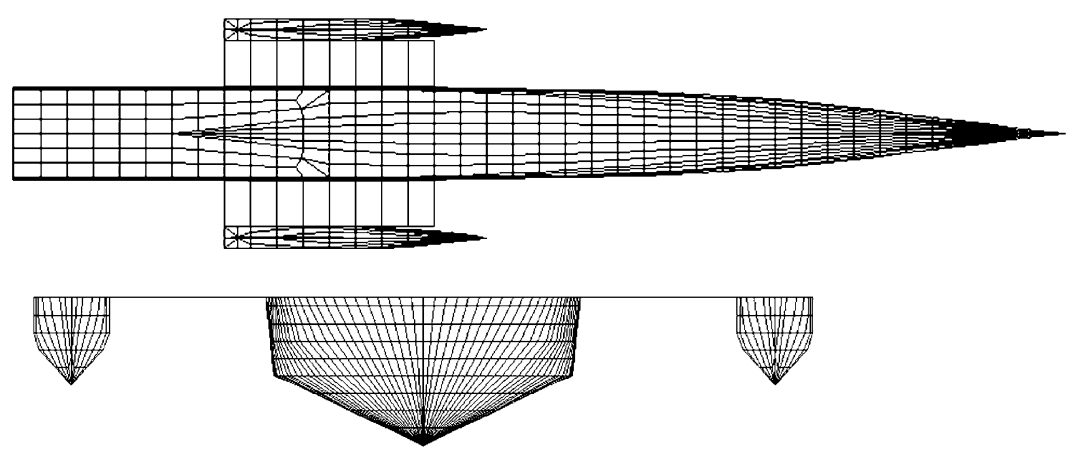

Fig. 8 Panel model with lid

If the lid was free to move without any external restraints, it could not provide any assistance to reduce the large wave elevation. Thereby, a fictitious linear damping coefficient $\mu_{p}$ was artificially added on the lid to suppress its motion induced by waves. An appropriate value of linear damping coefficient could be evaluated from experimental data. Tan et al. [24] also provided an equation to estimate the linear damping coefficient 


$$
\mu_{p}=\varsigma \frac{\omega_{n}^{3} \eta_{A}}{2 g}
$$

where:

$\varsigma=$ the non-dimensional energy loss coefficient

$\eta_{A}=$ the response amplitude of wave elevation

$\omega_{n}=$ the occurring frequency of large wave elevation

By using the lid approach, the impact of large elevation in the gap could be successfully suppressed, as shown in Fig. 9, where the prediction agreed with the experiment. Figure 10 presents the added mass and radiation damping coefficient calculated by the model with and without lid. The two added mass curves matched well except in the resonant region, where the added mass calculated from the model with lid was greater and the dent was filled. Furthermore, the radiation damping was approximately three times larger than that from the model without lid at the occurring frequency. The larger added mass and radiation damping could be a factor that reduces the heave motion at the occurring frequency. It should be noted that the radiation damping from lid model was apparently smaller before the occurring frequency. That might be caused by the lid because the lid suppressed the waves induced by the motions of hull which was directly related to the radiation damping.

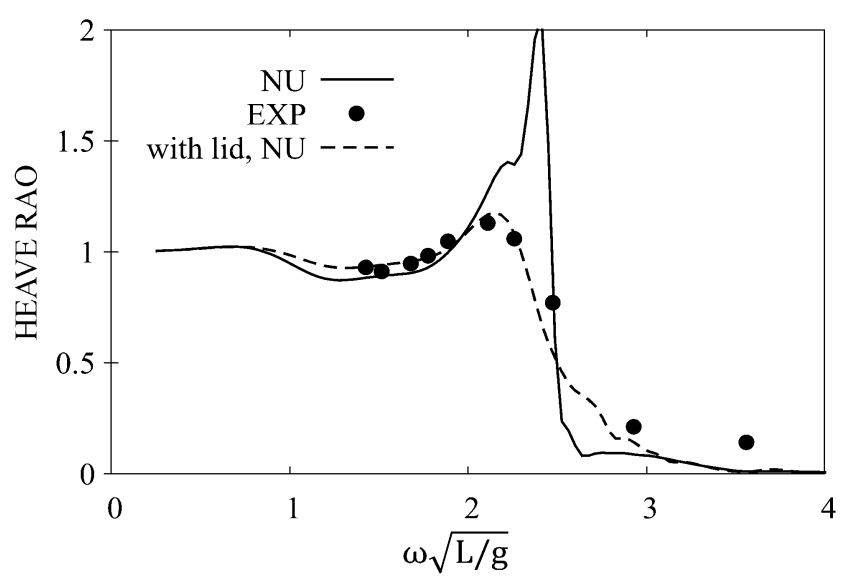

Fig. 9 Heave RAO deriving from the lid approach, 4-13, Fn=0.49

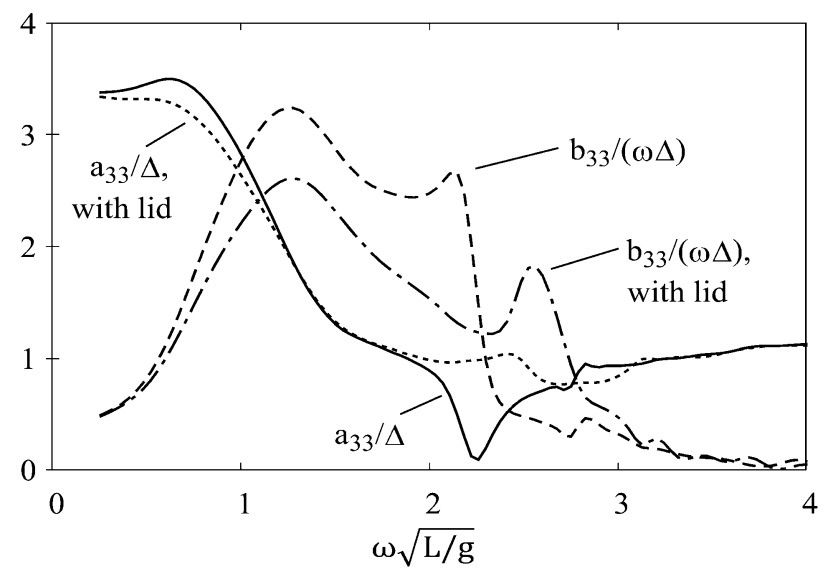

Fig. 10 Added mass and radiation damping for heave deriving from the lid approach, 4-13, Fn=0.49 


\subsection{The validation of lid approach}

A series of calculations for Scheme 4-07 and Scheme 4-13 were carried out and they were compared with the experimental data to verify the feasibility of the lid approach.

The RAOs of heave and pitch are nondimensionalized with respect to wave amplitude and wave number

$$
\begin{aligned}
& \text { Heave RAO: } H_{z}(\omega)=\frac{z_{a}}{\zeta_{a}} \\
& \text { Pitch RAO: } H_{\theta}(\omega)=\frac{\theta_{a}}{k \zeta_{a}}
\end{aligned}
$$

where:

$$
\begin{aligned}
& \omega=\text { circular frequency of incoming wave in } \mathrm{rad} / \mathrm{s} \\
& z_{a}=\text { amplitude of heave motion in } \mathrm{m} \\
& \theta_{a}=\text { amplitude of pitch motion in } \mathrm{rad} \\
& \zeta_{a}=\text { amplitude of incoming wave in } \mathrm{m} \\
& k=\text { wave number in } \mathrm{m}^{-1}
\end{aligned}
$$

Figure 11 and 12 illustrate the heave and pitch RAOs of Scheme 4-07 and 4-13 obtained from calculation and experiment, respectively. As shown in Fig. 11 and 12, most RAO curves calculated by the model without lid had two peaks. One reflected the trait of wave induced motions of the hull, which agreed with the tests. The other was induced by the unrealistic wave elevation in the gap, which deviated from the tests. In this peak region, the value of RAO obtained from the model without lid was irrationally larger than those obtained from the tests while the RAO obtained from the model with lid satisfactorily agreed with the RAO from tests. This demonstrated that the lid approach was able to practically deal with the issue with successful suppression of unrealistic wave elevation in between the main-hull and the outriggers. 
Zongyu Jiang, Yun Gao, Jie Liu
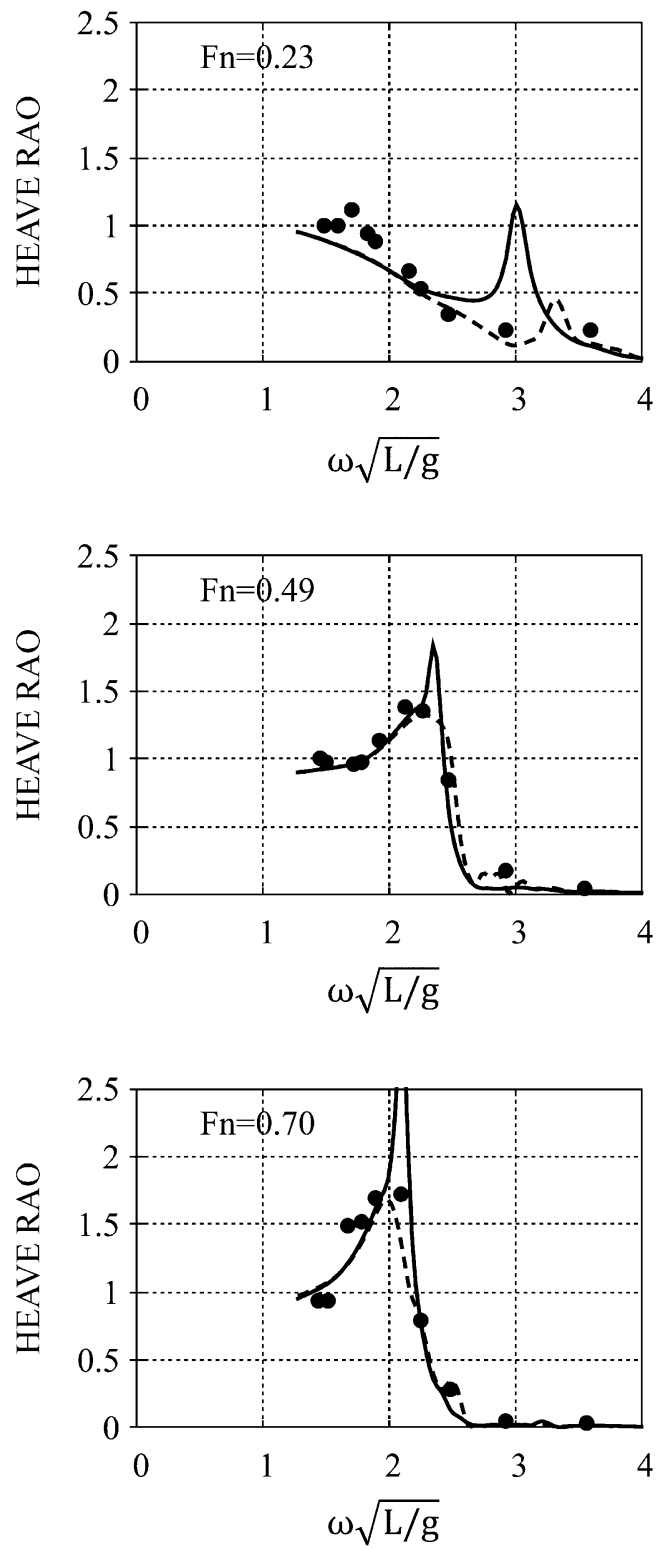

A lid approach for predicting wave induced motions of trimaran in regular waves
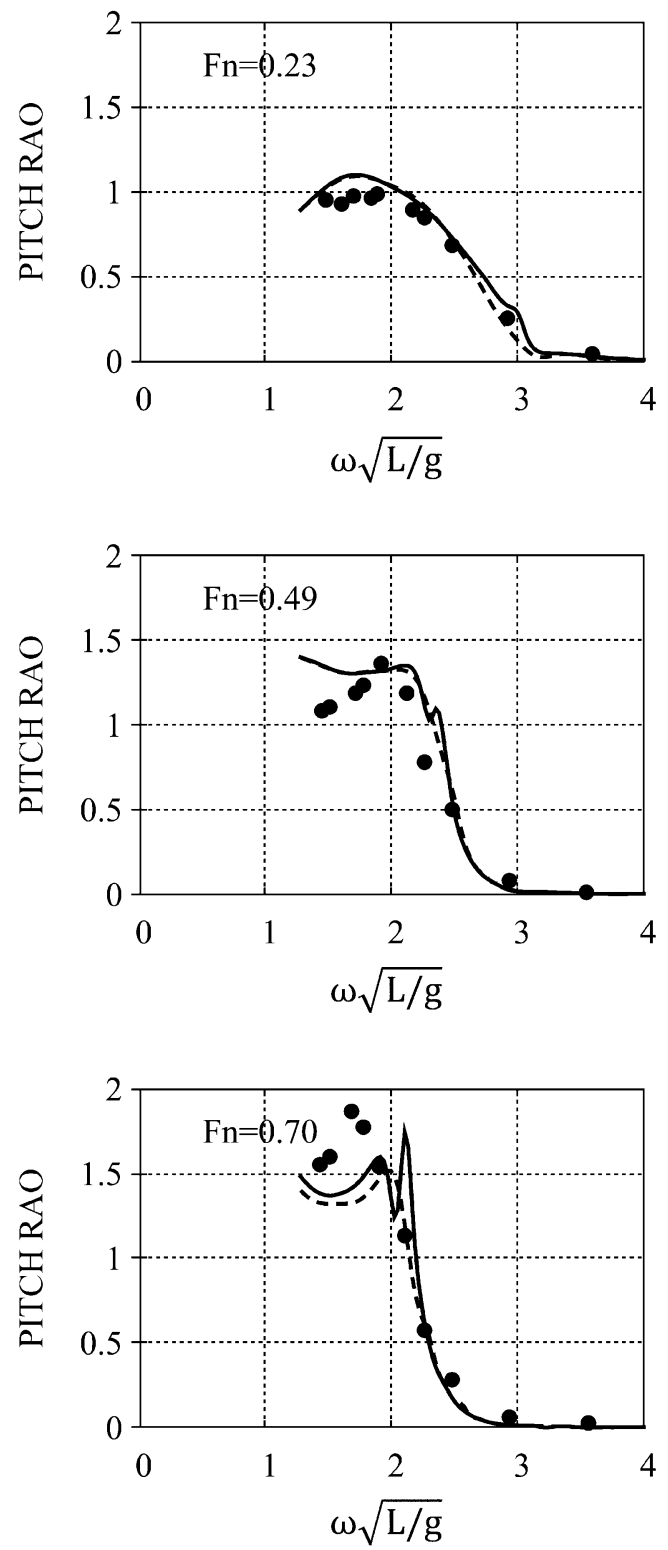

Fig. 11 Heave RAO and pitch RAO of Scheme 4-07. Dot: test; Solid line: numerical model without lid; Dashed line: numerical model with lid. 

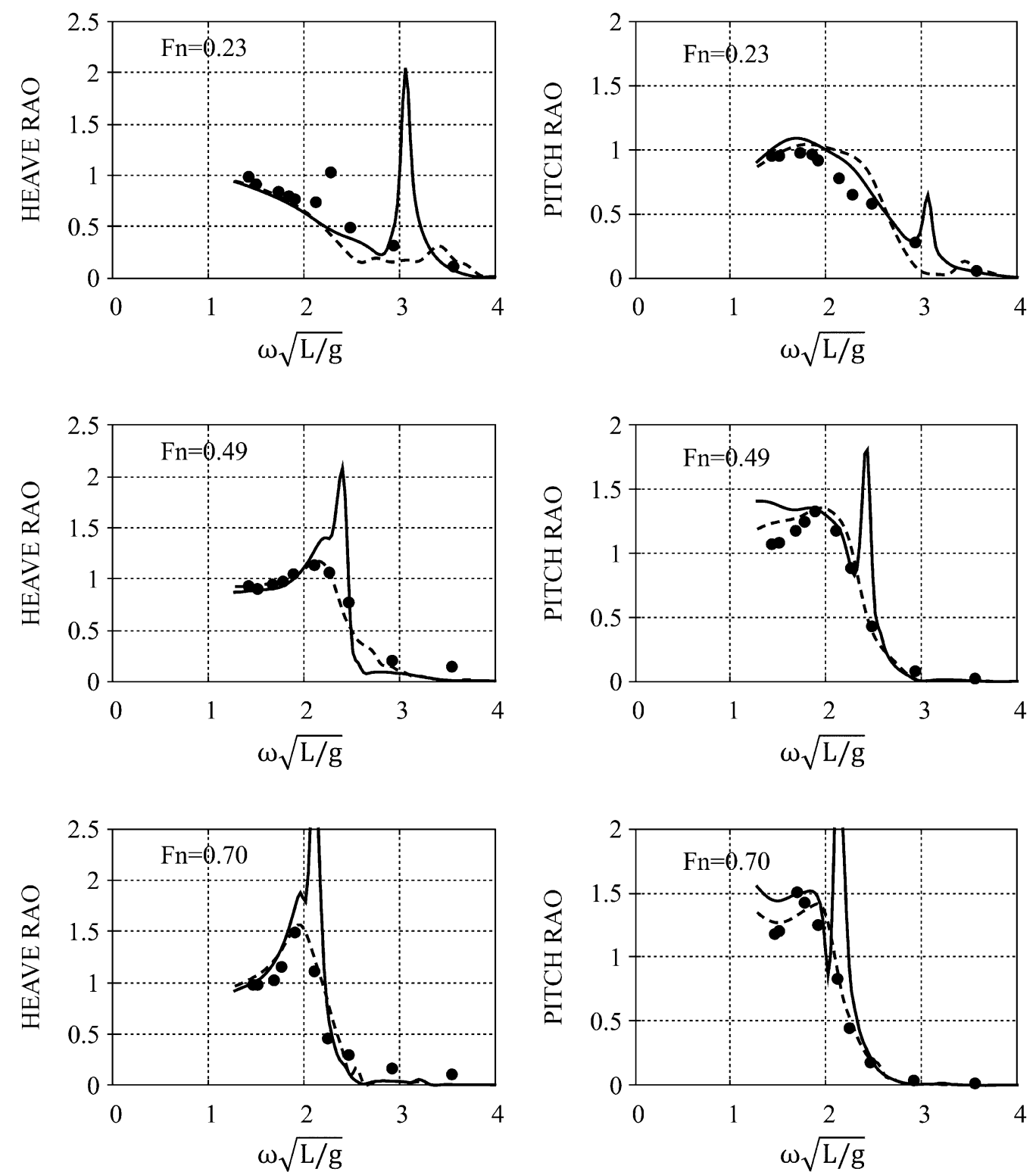

Fig. 12 Heave RAO and pitch RAO of Scheme 4-13. Dot: test; Solid line: numerical model without lid; Dashed line: numerical model with lid.

\section{Conclusion}

The wave induced motions of the trimaran were predicted by using the boundary integral method (BIM). The predicted wave elevation in between the trimaran's main-hull and outriggers were unrealistically large. However, the large wave elevation was not discovered during tests. The large wave elevation could induce large heave and pitch motions of the trimaran, which were also larger than the experimental results. The study revealed the equation raised by Molin could be used to predict the occurring frequency of the large wave elevation.

A practical approach was employed to suppress the large wave elevation by laying a lid on the free surface in between hulls. By using the lid approach, the large wave elevation was successfully suppressed. Meanwhile, this approach also suppressed the large motions induced 
by the large wave elevation. According to comparison with the test results, it was verified that the lid approach was able to practically predict motions of the trimaran sailing in regular waves.

\section{REFERENCES}

[1] Grassman J.M., and Hildstrom G.A., 2003. "Structural Trials of the RV Triton - a Status Update and Quick-Look Report."

[2] Bingham A.E., Hampshire J.K., Miao S.H., and Temarel P., 2001. «Motions and loads of a trimaran traveling in regular waves." Southampton: 6th International Conference on Fast Sea Transportation, 2001, 167-176.

[3] Jiao J.L., Sun S.Z., and Ren, H.L., 2016. "Prediction of wave induced ship motions and loads by largescale model measurement at sea and numerical analysis." BRODOGRADNJA, 67(2), 81-100. https://doi.org/10.21278/brod67206

[4] Hebblewhite K., Sahoo P.K., and Doctors L.J., 2007. "A case study: theoretical and experimental analysis of motion characteristics of a trimaran hull form." Ship and Offshore Structures, vol. 2, no. 2, 149-156. https://doi.org/10.1080/17445300701430242

[5] McDonald T.P., Bucknall R.W.G., and Greig A.R., 2013. "Comparing Trimaran Small Waterplane Area Center Hull (TriSWACH), Monohull, and Trimaran Hullforms: Some Initial Results.” Journal of Ship Production and Design, vol. 29, No. 4, 211-220. https://doi.org/10.5957/JSPD.29.4.120056

[6] Pavkov M., and Morabito M., 2014. "Experimental Investigation of Trimaran Models in Shallow Water." Journal of Ship Production and Design, vol. 30, No. 2, 66-78. https://doi.org/10.5957/JSPD.30.2.130056

[7] Wang S., and Wahab R., 1971. "Heave oscillations of twin cylinders in a free surface." Journal of Ship Research, vol., 15, no. 1, 33-48.

[8] Miao G.P., Liu Y.Y., Li Y.L., Liu, Z.Y., and Zhu, L.P., 1997. “On false resonance in application of strip theory to motion estimation for catamarans." Shipbuilding of China, 137, 32-38.

[9] Buchner B., van Dijk A., and de Wilde J., 2001. "Numerical multiple-body simulation of side-by-side mooring to an FPSO." Stavanger: Proc. 11th ISOPE, 2001, 343-353.

[10] Newman J. N., 2004. "Progress in wave load computations on offshore structures." Vancouver: Oral presentation at OMAE'2004.

[11] Newman, J. N., 1977. Marine Hydrodynamics, MIT Press: Cambridge, USA. https://doi.org/10.7551/mitpress/4443.001.0001

[12] Faltinsen, O. M., 1991. Sea loads on ships and offshore structures, Cambridge University Press: Cambridge, UK.

[13] Hess, J., and Smith, A.M.O., 1964. "Calculation of nonlifting potential flow about arbitrary threedimensional bodies.” J. Ship Research, 8, 22-44.

[14] John, F., 1950. “On the motion of floating bodies, II. Comm.” Pure and Applied Mathematics, 3, pp. 45101. https://doi.org/10.1002/cpa.3160030106

[15] Ohmatsu, S., 1975. "On the Irregular Frequencies in the Theory of Oscillating Bodies in a Free Surface." Papers of Ship Research Institute, 48, 1-13.

[16] Malenica S., and Chen X.B., 1998. "On the irregular frequencies appearing in wave diffraction-radiation solutions.” International Journal of Offshore and Polar Engineering, vol., 8, no., 2, 110-114.

[17] Lee, C.H., Newman, J.N., and Zhu, X., 1996. "An extended boundary integral equation method for the removal of irregular frequency effects." International journal for numerical method in fluids. Vol. 23, 637-660. https://doi.org/10.1002/(SICI)1097-0363(19961015)23:7<637::AID-FLD437>3.3.CO;2-V

[18] Zhu, X., 1994. "Irregular frequency removal from the boundary integral equation for the wave-body problem.” MSc Thesis, Dept. of Ocean Engineering, MIT.

[19] Molin, B., 1993. "Second-order hydrodynamics applied to moored structures." 19th WEGEMT School, Nantes, 126-175.

[20] Senjanovic, I., Malenica, S., Tomasevic, S., and Tomic, M., 2008. "Hydroelastic aspects of large container ships." Proceedings of the ASME 27th International Conference on Offshore Mechanics and Arctic Engineering, Esoril.

[21] Molin, B., 1999. “On the piston mode in moonpools.” Proceedings of the 14th Int. Workshop on water waves \& floating bodies.

[22] Chen X. B., 2004. "Hydrodynamics in offshore and naval applications - Part I." Perth: 6th International Conference on Hydrodynamics (ICHD 2004), 1-28. https://doi.org/10.1201/b16815 
[23] Kim M.S., Jung K.H., and Park S.B., 2018. "Wave induced coupled motions and structural loads between two offshore floating structures in waves.” BRODOGRADNJA, 69(3), 149-173.

[24] Tan L., Tang G.Q., Zhou Z.B., Cheng L., Chen X. B., and Lv L., 2017. "Theoretical and numerical investigations of wave resonance between two floating bodies in close proximity." Journal of Hydrodynamics, 29(5): 805-816. https://doi.org/10.1016/S1001-6058(16)60792-8

Submitted: 22.11.2017. Z Zongyu Jiang ${ }^{1, *}$, jzongyu@ hotmail.com

Accepted: $\quad$ 16.05.2019. Jie Liu ${ }^{4}$, Lucy.Liu@mathworks.cn

${ }^{1}$ Wood Group, Shanghai 201206, China

${ }^{2}$ State Key Laboratory of Oil and Gas Reservoir Geology and Exploration, Southwest Petroleum University, Chengdu 610500, China

${ }^{3}$ Department of Mechanical Engineering, The University of Tokyo, Tokyo 113-8656, Japan

${ }^{4}$ MathWorks, Shanghai 200122, China

* Corresponding author (Zongyu Jiang): jzongyu@ hotmail.com 